\title{
Agrobacterium and viral vectors-mediated in vivo gene silencing assays in plants
}

\begin{abstract}
Over the last years, the explosive growth of plant genomic resources and the development of advanced vectors enhanced the emergence of rapid systems to dissect plant gene function. Although transgenic approach has been applied to generate stable transgenic lines, this procedure is time-consuming and labor-intensive. Transient expression systems are continuously emerging during these years as an alternative tool for functional genomics studies in plants. Among these techniques, Agrobacterium-infiltration and infectious clones-derived plant viruses have been developed and successfully applied for versatile transient assays. Their effectiveness over a wide range of plant species is often validated. Here, the green fluorescent protein (GFP) gene was investigated as reporter gene for proof-of-concept to induce gene silencing in GFP-transgenic tobacco and grapevine plants either by inoculation of Arabis mosaic virus infectious clones or through in planta vacuum infiltration of Agrobacterium suspension.
\end{abstract}

Leaves derived from greenhouse-grown grapevine plants were vacuum infiltrated with agrobacteria suspension containing GFP gene to induce silencing. Nicotiana benthamiana plants were agro-inoculated with Arabis mosaic virus infectious clones $\mathrm{RNA}_{1}$ and $\mathrm{RNA}_{2}$ along with a modified RNA satellite harboring a GFP construct. Systemic GFP-silencing was monitored during days and weeks after inoculation and data showed that silencing was achieved after 10-12 days post inoculation for tobacco, while it takes 6 weeks to be completely reached in grapevine leaves. The transient expression systems established here were successfully applied for inducing a knock-out of endogenous gene. These techniques provide a fast and reliable tool to carry out gene function analysis in plants and open a future outlook with several downstream applications towards pathogen resistance or abiotic stress tolerance.

Keywords: agro infiltration, agro-inoculation, viral infectious clones, transient expression system, virus induced gene silencing, arabis mosaic virus, viral vector, gfp silencing, tobacco, grapevine
Volume I Issue 2 - 2017

\author{
Anis Ben Amar,' Thierry Wetzel ${ }^{2}$ \\ 'Department of Plant Molecular Physiology, Center of \\ Biotechnology of Borj Cedria, Tunisia \\ 2Institute of Plant Protection, DLR-Rheinpfalz, Neustadt ander \\ Weinstrasse, Germany
}

Correspondence: Anis Ben Amar, Department of Plant Molecular Physiology, Center of Biotechnology of Borj Cedria, Tunisia, Tel 00216.26478256, Fax 0021679325638, Email anisbenamar.cbbc@gmail.com

Received: July 0I, 2017 | Published: September 12, 2017
Abbreviations: ArMV, arabis mosaic virus; DPI, day post infiltration; dsRNA, double stranded RNA; GFP, green fluorescent protein; PTGS, post-transcriptional gene silencing; VIGS, virusinduced gene silencing; WPI, week post infiltration

\section{Introduction}

Functional genomics could be investigated by over-expressing or silencing a gene sequence towards a better understanding of its biological function at different organisation level (plant cell, tissue, organ or even in the whole plant) in response to various situations (plant developmental stages, environmental stress, plant-pathogen interactions). Actually, several trends are focusing on transient expression systems without the need to produce stable transgenic plants. ${ }^{1,2}$ These non-transgenic approaches involve a battery of rapid and versatile transient assays mediated either by Agrobacterium tumefaciens or by viral-based vectors. ${ }^{3}$ Both of these systems have been extensively applied recently for high throughput reverse genetics and functional gene analysis in plants. ${ }^{4}$

\section{Agro inoculation of viral infectious clones}

During the last years, genome project have generated a wealth of genome sequence information. Currently, the future challenge is to accelerate the identification of the biological function of these genes. Viral vectors provide an attractive system to express a sequence of interest or to induce virus-induced gene silencing ${ }^{5}$ that turn into a common reverse genetics tool for functional studies in model plants. ${ }^{4-6}$ Numerous methods have been employed to initiate this homologybased RNA degradation process, but all rely on the activity of doublestranded RNAs (dsRNAs) corresponding to the gene of interest. Virusinduced gene silencing (VIGS) has gained acceptance as the tool of choice for transient induction of silencing. ${ }^{7}$ Since the first report on Agro-infection as an alternative route for viral inoculation in plants. ${ }^{8}$ recent significant advances in the construction of plant virus infectious clones in a binary vector intended for Agrobacterium inoculation was achieved. ${ }^{9}$ For instance, in case of grapevine, different viruses have been engineered and cDNA infectious clones of the Grapevine virus A (GVA), ${ }^{10}$ the Grapevine leaf roll-associated virus-2 (GLRaV-2) ${ }^{11}$ and the Grapevine rupestris stem pitting associated virus $(\mathrm{GRSPaV})^{12}$ have been constructed to express exogenous DNA and could be agroinoculated to plants.

The Nepovirus Arabis mosaic virus (ArMV), amongst Grapevine fan leaf virus (GFLV) and Grapevine leaf roll-associated virus (GRLaV) infecting grapevine by nematode transmission causing considerable yield losses. Nepoviruses have two positive sense, single stranded genomic RNAs, which are polyadenylated at their $3^{\prime}$ end $^{13}$ and have a covalently attached small genome-linked viral protein (VPg) at their $5^{\prime}$ end. ${ }^{14}$ The complete nucleotide sequences of $\mathrm{RNA}_{1}$ and $\mathrm{RNA}_{2}$ of the ArMV-NW (isolates Neustadt/Weinstrasse) 
from grapevine have been reported. ${ }^{15,16}$ Some isolates of ArMV have a small genomic RNA called RNA satellite, whose full-length sequence has been determined. ${ }^{17}$ The ArMV-NW infectious clones can be inoculated onto Chenopodium quinoa and retro-inoculated onto Nicotiana benthamiana resulting in a systemic infection. These viral infectious clones, as well as the RNA-satellite, provide potential vectors for molecular cloning and gene expression studies. In the present study, viral RNA satellite was modified by insertion of GFP to be used as viral vector.

For this purpose, ArMV RNA-satellite was cloned into the pCass2 vector (Figure 1a). Since the discovery of visual reporter protein like the Green fluorescent protein (GFP) which could be expressed in plants as gene fusion; such gene coding for fluorescent protein has been extensively used. ${ }^{18}$ In this regard, GFP gene was cloned into the RNAsatellite of the ArMV that we developed as a viral vector of foreign gene (Figure 1). Briefly, a cloning strategy based on extension PCR was performed to induce a mutation and create a BamHI restriction site into the satellite clone (data not shown). GFP construct of 700pb was amplified by PCR (GeneAmp9700, Applied Biosystems, USA) using specific primers and introduced into BamHI cloning site into the ArMV-satellite clone. GFP-engineered ArMV satellite construct was assessed by PCR amplification (Figure 1b) as well as northernblot hybridization (Figure 1c). Satellite clones were introduced into Agrobacterium for agro-inoculation purpose. N. Benthamiana plants were grown in greenhouse and agro-inoculated simultaneously with the GFP-satellite (Sat: GFP) in the presence of viral infectious clones (ArMV-RNA 1 and - $\mathrm{RNA}_{2}$ ) as helper virus. GFP expression assay was monitored using a hand-held long-wavelength ultraviolet lamp.

Experiments were carried out using 5 replicates in 3 independent experiments. ELISA test using monoclonal specific-antibody against ArMV revealed that 80 to $90 \%$ of the inoculated host plants were infected. Positive ELISA samples were used for RNA extraction by GENOS RNA extraction and purification kit. Northern blot hybridization was performed with non-radioactive DIG method (Roche Applied Science) and confirmed the presence of the satellite as modified with the GFP construct, compared to RNA from native satellite used as control (Figure 1c). In this study, GFP-engineered satellite was firstly applied to investigate GFP expression in wild type (WT) N. Benthamiana plants after agro-inoculation of ArMV clones on tobacco leaves (Figures 1d-1f). Green fluorescence was visible under UV light since 3dpi on agro-inoculated leaves until it covered the whole area of the leaves within 7dpi (Figure 1f) compared to WT tobacco plants (Figure 1d) (Figure1e). The ArMV-RNA-satellite modified by insertion of GFP gene construct has been successfully transmitted during virus replication.

In this study, the ArMV satellite has been modified with gene encoding GFP as visual marker. A modified Tobacco rattle virusGFP vector has been also produced recently for efficient and visualizable analysis of gene function. ${ }^{19}$ For a GLRaV-2-derived vector, systemic gene expression was detectable in grapevine from 4weeks post inoculation. ${ }^{11}$ It was established that expression occurred exclusively at the RNA level and infected plants still expressed the exogenous sequence up to 15 months post inoculation. Furthermore, GFP silencing in transgenic 16-C GFP-expressing N. Benthamiana plants was investigated (Figures 2a-2c). Transgenic tobacco plants constitutively expressing GFP: line 16C (Figure 2a) were coinoculated with GFP-modified ArMV satellite along with agrobacteria containing ArMV-RNA 1 and ArMV-RNA 2 and showed slight reduction of their fluorescence within the first 4-6dpi (Figure 2b). After that, the silencing progressively moved to the non-inoculated areas and extended at systemic level showing complete virus-induced GFP silencing 10-12 dpi (Figure 2c) compared to the control noninoculated plants.

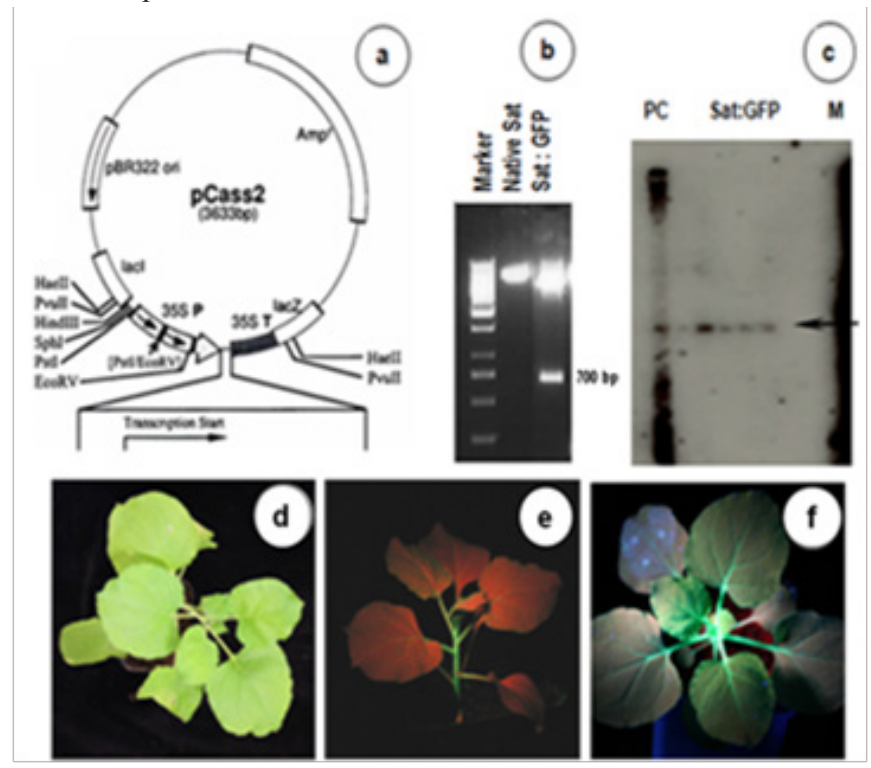

Figure I Construction of GFP-engineered ArMV satellite viral clone (upper panel) and transient agro-inoculation assays on Nicotiana benthamiana plants (down panel).

a. PCass2 vector used for cloning genomic RNA of ArMV to produce viral infectious clones (full length clones of virus RNA, and RNA, as well as the sub viral RNA).

b. PCR amplification of the viral satellite clone modified or not with GFP insertion into BamHI cloning site. Digestion profile with BamHI gives a fragment of about 700 bp corresponding to the GFP.

c. Northern blot hybridization for detection of GFP expression in agro-inoculated tobacco plants showing high level of GFP transcripts deriving from the modified ArMV sub viral satellite clone used as vector for foreign gene expression and virus-induced gene silencing.

d. The down panel illustrates transient GFP expression following agro-inoculating GFP-satellite on tobacco leaves. Photography of WT Nicotiana benthamiana plant under normal (a) and UV light (b) showing no fluorescence. Green fluorescence appears on newly developed leaves within 4 days post inoculation (c).

e. M, Marker; PC, Positive Control; Native Sat, Non-Modified ArMV SateIlite Clone Sat; GFP, GFP-Engineered ArMV Satellite Clone

A virus-induced gene silencing (VIGS) mechanism is thus launched upon agro-inoculation of tobacco plants with those agrobacteria strains by accumulation of GFP transcripts that triggers an RNA interference, a process by which dsRNA induces the gene silencing. In fact, upon infection of a plant host usually via Agrobacterium tumefaciens, this recombinant viral vector induces post transcriptional gene silencing (PTGS) targeting both the virus RNA and homologous endogenous plant RNA sequences for degradation..$^{20}$ While several plant viruses have been so far developed into VIGS vectors, the Tobacco Rattle Virus (TRV) established by Baulcombe's group has been shown to provide the most robust results in terms of efficiency and ease of application. ${ }^{21}$ It is widely known that VIGS can occur for about 3 weeks, and in few cases the duration of VIGS can be prolonged for 
up to 3 months..$^{22}$ So far, it has been stated that inoculated plants by viral vectors still expressed the exogenous sequence up to 15 months ${ }^{11}$ and in other report, VIGS can persist for more than 2years and also be transmitted to progeny seedlings in tobacco and tomato. ${ }^{22}$ Our data showed that green fluorescence present at systemic level should reflect that the satellite replication was in correlation with the movement of the virus though the entire plant. Further studies are currently on the way to investigate the application of such viral system for inducing endogenous gene silencing in grapevine using the VIGS strategy, whose provide a powerful option for functional studies. In a similar way, small sub viral RNA satellite along with helper-virus dependant replication were used recently as other source of VIGS vectors to induce silencing. ${ }^{23}$

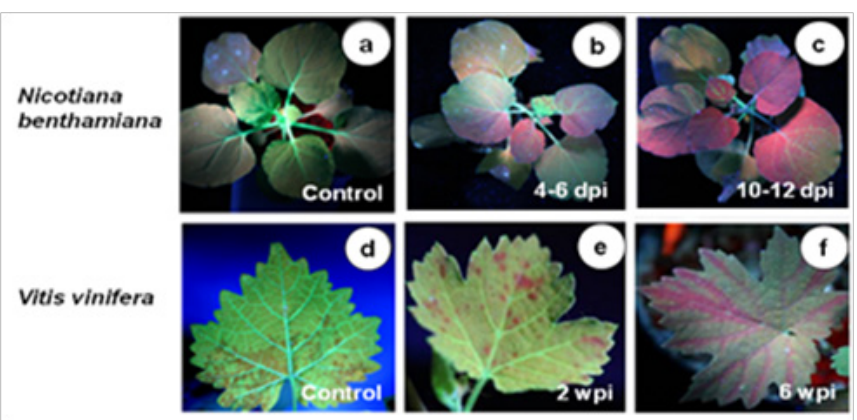

Figure 2 Silencing assay of constitutive GFP expression in GFP-transgenic plants: Nicotiana benthamiana (upper panel) and Vitis vinifera (bottom panel). GFP-expressing tobacco line 16C.

a. Agro-inoculated with GFP-engineered ArMV-satellite to induce VIGS that started since 4-6 days post inoculation showing reduction of fluorescence.

b. Complete silencing within I0-12 DPI.

c. Similarly, GFP-transgenic grapevine leaves.

d. Were subjected to vacuum infiltration of Agrobacterium tumefaciens strain GV3I03 harboring 35S: GFP. GFP silencing after 2 and 6weeks post infiltration was observed, respectively.

e. Photography was taken using a hand-held long-wavelength ultraviolet lamp to allow fluorescence detection. Each picture is representative of 5 agro-inoculated plants.

Promising results described that genes involved in abiotic stress tolerance can be analyzed through a virus-based vectors. ${ }^{24}$ For instance, tobacco plants showed enhanced salinity and heat tolerance when agro-inoculated leaves transiently expressing a tomato phospholipid hydroperoxide glutathione peroxidase (LePHGPx) gene cloned in a PVX-based vector. ${ }^{25}$ Furthermore, a rapid analysis to determine the role of plant promoters and transcription factors in abiotic stresses is possible by simple agro-infiltration of the appropriate plasmid constructs into tobacco leaves. ${ }^{26}$ However, the use of viral vectors for functional analysis has not yet been reported in grapevine. ${ }^{27}$

\section{Agro-infiltration based system for silencing studies}

Besides using the potential of virus-based vectors to inoculate plants, transient expression can be carried out using Agrobacterium infiltration, also known as agro-infiltration, which generally involves a bacterial suspension that will be forced to infiltrate the leaf stomata by needless syringe. ${ }^{1}$ This method is fast, simple and widely used in tobacco and solanaceae plants, but tends to restrict gene expression to the infiltration zones. Moreover, the feasibility of agro-infiltration in grapevine is limited to young in vitro leaves. ${ }^{1-28}$ In previous study ${ }^{2}$ we established a vacuum infiltration system able to infiltrate grapevine leaf in planta for transient expression assays. The method consists of plunging leaves or whole plants into the bacterial suspension and then transiently applying a vacuum pressure to facilitate liquid penetration into the mesophyll cells. Contrary to the syringe infiltration method, vacuum infiltration allows gene expression in the whole leaf..$^{27}$ Recent attempts using the GFP as fluorescent marker for grapevine functional genomics were achieved by in situ vacuum agro-infiltration. ${ }^{2}$

Here, we propose this system as a reliable option for silencing assays in grapevine. In practice, Agrobacterium suspension containing a binary vector harboring the GFP was vacuum infiltrated into plant leaves of transgenic greenhouse-grown grapevine plants stably expressing the GFP protein. Introduction of T-DNA holding the GFP will cause a post-transcriptional gene silencing mechanism that triggers the GFP transcripts inducing a knockout of GFP with loss of green fluorescence. Non-detached leaves from green cuttings GFPtransgenic lines of grapevine (Vitis vinifera) (Figure 2d) were used as the target organ and vacuum infiltrated as described before ${ }^{2}$ for in planta inoculation with $A$. Tumefaciens harboring GFP gene construct as visual reporter gene. Evidence of GFP silencing in transgenic GFP-expressing grapevine via agro-infiltration was reported since 2 weeks post infiltration (Figure 2e) with decreased fluorescence over the time until reaching complete silencing within 6weeks (Figure 2f). We observed that target gene silencing in the newly developed noninoculated leaves occurs faster in tobacco than in grapevine. While agro-infiltration method allows transient gene expression in plants, the viral-based vector relies on the replication of viral genomic RNA as long as the host plant is still alive, providing stable expression of the introduced gene in the infected plant cells. Taking together, both agro-infiltration and viral clones-inoculation have several applications in modern plant molecular biology relaying on reverse genetics-based functional genomics. ${ }^{4}$

\section{Conclusion}

There is considerable interest in such rapid assays for assigning gene function, especially in case where some plant species are recalcitrant to in vitro regeneration and to generation of stable transgenic lines. These alternative systems involving an agroinfiltration of modified agrobacteria strains or an agro-inoculation of virus infectious clones as vehicles for foreign gene expression/ silencing emerged as versatile tools suitable for functional analysis. Such established methods described here were successfully applied not only in model plants like tobacco, but also in case of grapevine plants with significant efficiency. We demonstrated the feasibility of using agro-inoculation-based viral vectors for transient assay and virus-induced gene silencing in $N$. Benthamiana along with the efficiency of gene silencing-based agro-infiltration in grapevine using GFP reporter gene. These systems could be applied to express and silence genes related to fungal/viral resistance or analysis of abiotic stress-induced genes from heterologous species towards functional genomics and plant genetic improvement.

\section{Conflict of interest}

The author declares no conflict of interest.

\section{Acknowledgements}

This work was supported by the Tunisian Ministry of Higher 
Education \& Scientific Research and partially funded in the frame of the Tunisian-German Cooperation in Science and Technology (TUNGER-2015-32).

\section{References}

1. Zottini M, Barizza E, Costa A, et al. Agroinfiltration of grapevine leaves for fast transient assays of gene expression and for long-term production of stable transformed cells. Plant Cell Rep. 2008;27(5):845-853.

2. Ben Amar A, Cobanov P, Buchholz G, et al. In planta agroinfiltration system for transient gene expression in grapevine. Acta Physiol Plant. 2013;35(11):3147-3156.

3. Burch Smith TM, Anderson JC, Martin GB, et al. Applications and advantages of virus-induced gene silencing for gene function studies in plants. Plant J. 2004;39(5):734-746.

4. Ben Amar A, Daldoul S, Reustle GM, et al. Reverse genetics and high throughput sequencing methodologies for plant functional genomics. Curr Genomics. 2016;17(6):460-475.

5. Becker A, Lange M. VIGS-genomics goes functional. Trends Plant Sci. 2010;15(1):1-4.

6. Huang C, Qian Y, Li Z, et al. Virus-induced gene silencing and its application in plant functional genomics. Sci China Life Sci. 2012;55(2):99-108.

7. Padmanabhan M, Dinesh Kumar SP. Virus-induced gene silencing as a tool for delivery of dsRNA into plants. Cold Spring Harbor Protoc. 2009;2(4):1-4.

8. Blawid R, Nagata T. Construction of an infectious clone of a Plant RNA virus in a binary vector using one step Gibson Assembly. J Virol Methods. 2015;222:11-15.

9. Muruganantham M, Moskovitz Y, Haviv S, et al. Grapevine virusA-mediated gene silencing in Nicotiana benthamiana and Vitis vinifera. J Virol Methods. 2009;155(2):167-174.

10. Kurth EG, Peremyslov VV, Prokhnevsky AI, et al. Virus derived gene expression and RNA interference vector for grapevine. $J$ Virol. 2012;86(2):6002-6009.

11. Meng B, Venkataraman S, Li C, et al. Construction and biological activities of the first infectious cDNA clones of the genus Foveavirus. Virology. 2013;435(2):453-462.

12. Ben Amar A, Bassler A, Ghorbel A, et al. Determination of the length of the poly (A) tails of the Arabis mosaic nepovirus genomic RNAs. Plant Viruses. 2008;1(2):208-210.

13. Mayo MA, Robinson DJ. Nepoviruses: molecular biology and replication. In: Harrison, Murant, editors. The Plant viruses, Polyedral virions and bipartite RNA genomes. New York, USA: Plenum Press; 1996.

14. Wetzel T, Meunier L, Jaeger U, et al. Complete nucleotide sequence of the RNAs 2 of german isolates of Grapevine fanleaf and Arabis mosaic nepoviruses. Virus Res. 2001;149(5):139-145.
15. Wetzel T, Beck A, Wegener U, et al. Complete nucleotide sequence of the RNA 1 of a grapevine isolate of Arabis mosaic virus. Arch Virol. 2004;149:989-995.

16. Wetzel T, Bassler A, Amren MAW, et al. A RT/PCR-partial restriction enzymatic mapping (PREM) method for the molecular characterization of the large satellite of Arabis mosaic virus. J Virol Meth. 2006;132(1-2):97-103.

17. Ryu CM, Anand A, Kang L, et al. Agrodrench: a novel and effective agroinoculation method for virus-induced gene silencing in roots and diverse Solanaceous species. Plant J. 2004;40(2):322-331.

18. Nurkiyanova KM, Ryabov EV, Commandeur U, et al. Tagging Potato leaf roll virus with the jellyfish green fluorescent protein gene. $J$ Gen Virol. 2000;81(pt 3):617-626.

19. Tian J, Pei H, Zhang S, et al. TRV-GFP: a modified Tobacco rattle virus vector for efficient and visualizable analysis of gene function. $J$ Exp Bot. 2014;65(1):311-322.

20. Senthil Kumar M, Mysore KS. Tobacco rattle virus-based virus-induced gene silencing in Nicotiana benthamiana. Nature Protoc. 2014;9(7):1549-1562.

21. Senthil Kumar M, Mysore KS. Virus-induced gene silencing can persist for more than 2 years and also be transmitted to progeny seedlings in $\mathrm{Ni}$ cotiana benthamiana and tomato. Plant Biotechnol J. 2011;9(7):797-806.

22. Cai X, Wang $\mathrm{C}, \mathrm{Xu} \mathrm{Y}$, et al. Efficient gene silencing induction in tomato by a viral satellite DNA vector. Virus Res. 2007;125(2):169-175.

23. Senthil Kumar M, Rame Gowda HV, Hema R, et al. Virus-induced gene silencing and its application in characterizing genes involved in waterdeficit-stress tolerance. J Plant Physiol. 2008;165(13):1404-1421.

24. Chen S, Vaghchhipawala Z, Li W, et al. Tomato phospholipid hydroperoxide glutathione peroxidase inhibits cell death induced by Bax and oxidative stresses in yeast and plants. Plant Physiol. 2004;135(3):1630-1641.

25. Yang Y, Li R, Qi M. In vivo analysis of plant promoters and transcription factors by agroinfiltration of tobacco leaves. Plant J. 2000;22(6):543-551.

26. Jelly NS, Valat L, Walter B, et al. Transient expression assays in grapevine: a step towards genetic improvement. Plant Biotechnol J. 2014;12(9):1231-1245.

27. Santos Rosa M, Poutaraud A, Merdinoglu D, et al. Development of a transient expression system in grapevine via agro-infiltration. Plant Cell Rep. 2008;27(6):1053-1063.

28. Grimsley N, Hohn B, Hohn T, et al. Agroinfection, an alternative route for viral infection of plants by using the Ti plasmid. Proc Natl Acad Sci USA. 1986;83(10):3282-3286. 